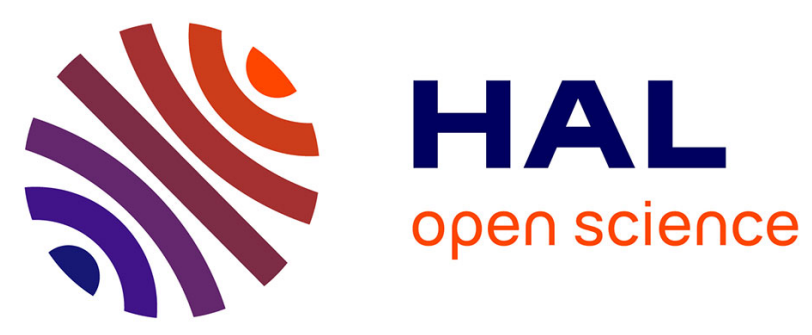

\title{
Reproducible formation of single magnetic bubbles in an array of patterned dots
}

Tao Liu, Vito Puliafito, Francois Montaigne, S. Petit-Watelot, Cyrile Deranlot, Stéphane Andrieu, Ozhan Ozatay, Giovanni Finocchio, Thomas Hauet

\section{- To cite this version:}

Tao Liu, Vito Puliafito, Francois Montaigne, S. Petit-Watelot, Cyrile Deranlot, et al.. Reproducible formation of single magnetic bubbles in an array of patterned dots. Journal of Physics D: Applied Physics, 2016, 10.1088/0022-3727/49/24/245002 . hal-01345414

\author{
HAL Id: hal-01345414 \\ https://hal.science/hal-01345414
}

Submitted on 18 Jul 2016

HAL is a multi-disciplinary open access archive for the deposit and dissemination of scientific research documents, whether they are published or not. The documents may come from teaching and research institutions in France or abroad, or from public or private research centers.
L'archive ouverte pluridisciplinaire HAL, est destinée au dépôt et à la diffusion de documents scientifiques de niveau recherche, publiés ou non, émanant des établissements d'enseignement et de recherche français ou étrangers, des laboratoires publics ou privés.

\section{(ㅇ)(1) $\$$}

Distributed under a Creative Commons Attribution - NonCommercial - NoDerivatives| 4.0 


\title{
Reproducible formation of single magnetic bubbles in array of patterneddots
}

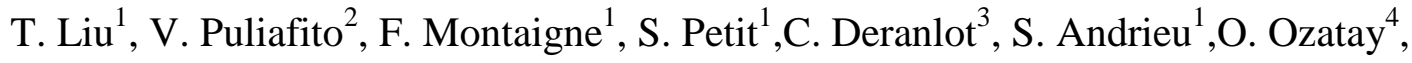 \\ G. Finocchio ${ }^{2}$ and T. Hauet ${ }^{1}$ \\ ${ }^{1}$ Institut Jean Lamour, UMR CNRS-Université de Lorraine, 54506 Vandoeuvrelès Nancy, France \\ ${ }^{2}$ Department of Engineering, University of Messina, C.da Di Dio, I-98166 Messina, Italy \\ ${ }^{3}$ Unité Mixte de Physique CNRS/Thales, associée à l'Université Paris-Sud, 91767 Palaiseau cedex, France \\ ${ }^{4}$ Department of Physics, BogaziciUniversity, 34342 Bebek/Istanbul, Turkey
}

E-mail: thomas.hauet@univ-lorraine.fr

\begin{abstract}
Formation conditions of single magnetic bubblesthrough in-plane field demagnetization are investigated in an array of Co/Ni circular dots by magnetic force microscopy and compared to micromagnetic calculations. We demonstrate high success rates in nucleating stable bubbles. The efficiency of single bubble formation is found to depend not only on the dot size, material thickness and intrinsic material parameters but also on the bubble nucleation path. Experimental phase diagrams and micromagnetic calculations highlightthe influence of the starting in-plane field amplitude and dipolar interactionsin stabilizing the bubble. The identification of a systematic procedure for controlling nucleation of single bubbles,multidomain states or a uniform state is important from a technological point of view opening a path toward the realization of reprogrammable magnonic crystals for the control of spin-wave propagation.
\end{abstract}

Keywords: magnetic bubbles, perpendicular magnetic anisotropy, magnetic force microscopy, micromagnetic simulations 


\section{INTRODUCTION}

Several numerical analyses and theoretical studies have recently predicted that magnetic soliton states observed in systems with perpendicular magnetic anisotropy (PMA), i.e. bubbles andskyrmions, would have the potential to significantly widen the scope ofrecording and dynamics of existing spintronic devices[1,2,3, 4,5].

Magnetic bubbles, in particular, are spots of opposite magnetization in an otherwise uniformly magnetized perpendicular anisotropy magnetic thin filmeither with zero[6, 7]or integer topological charge [8], the latter being recently called skyrmion bubbles $[9,10]$. On the other hand, skyrmions, characterized by a topological number equal to \pm 1 , were initially predicted $[3$, $11,12]$ and finally observed $[13,14]$ in thin ferromagnetic films coupled to heavy metals where the interfacial Dzyaloshinskii-Moriya interaction (DMI) $[15,16]$ plays a decisive role (differently from skyrmion bubbles that are due to a trade-off between exchange and dipolar field [9]).

Experimentally, bubbles wereinduced in several extended thin films, by external magnetic field[10, 17], by local magnetic field from a magnetic tip [18], by locally injecting spin polarized current in nano-contacts [19, 20], or in antiferromagnetic coupled multilayers [21]. There are fewerreports on forming a bubble in confined geometry, i.e. in microor nano-structured films. Appearance of a bubble was demonstrated in micrometer-sizedcylindrical Co [22], Permalloy[23], $\mathrm{Ni}$ [24] dots with weak PMA, in circular dots of $\mathrm{L}_{0} \mathrm{FePt}$ dots with large PMA energy[25].The last work, in particular, studiedan array of dots thatwas initially saturated by means of a large perpendicular field, then, at remanence, less than $15 \%$ of dots were in a bubble state.Overcoming this lack of reproducibility would give hope in achieving both spin-transfer-torque (STT)-based devices analogous to the vortex based nano-oscillators [3,26, 27, 28]and original magnonic crystals.

In this paper, we report a procedurethat enablesreproduciblenucleation ofwell-definedsingle magnetic bubbles in confined dots. The experiments have been performedon an assemblyof $\mathrm{Co} / \mathrm{Ni}$ circular dots with diameters ranging from 4 micron down to $200 \mathrm{~nm}$, and with thicknesses from 20 to 7 repeats (i.e. $16 \mathrm{nmdown}$ to $5 \mathrm{~nm}$ ). DMI is here negligible for two main reasons, (i) the large thickness of the ferromagnet [3] and the coupling to the same heavy metal (Pt) both in the lower and upper interface of the ferromagnet. Micromagnetic simulationsare usedto qualitativelyunderstand the mechanism of bubble formation andto identify the key ingredientsthat rule the reproducible magnetic bubble nucleation.

The paper is organized as follows. Section 2 provides the details of the experimentalframework. Results and discussions are given in Section 3, whileSection 4 is dedicated to the conclusions.

\section{EXPERIMENTAL DETAILS}


$\mathrm{Co} / \mathrm{Ni}$ multilayer is being considered as a good candidate in view of STT applications providing several ofthe requested features such as low damping, high perpendicular anisotropy, high spin-polarization and moderate magnetization[29, 30, 31, 32, 33]. A set of $\operatorname{Ta}(5) / \mathrm{Pt}(10) /[\mathrm{Co}(0.2) / \mathrm{Ni}(0.6)]_{\mathrm{n}=7,10,15,20} / \mathrm{Pt}(5)($ where the numbers in parentheses are the nominal thickness of the individual layers in nanometers) multilayer was deposited on thermally oxidized Si wafers at room temperature by magnetronsputtering. The magnetic properties of the fullfilms at room temperature were determined by SQUID-VSM. All multilayer films have saturation magnetization $M_{\mathrm{S}}$ around500 $\pm 50 \mathrm{kA} / \mathrm{m}$ and PMAK, measured as the in-plane saturation field, around $3.6 \pm 0.9 \times 10^{5} \mathrm{~J} / \mathrm{m}^{3}$.

$\mathrm{The}[\mathrm{Co} / \mathrm{Ni}]_{\mathrm{n}}$ films were patterned to circular dots, withdiameters ranging from 0.2 to $4 \mu \mathrm{m}$, by combining electron beam lithography and ion beam etching. The distance between dots (center-to-center)was set to be four times their diameter in order to have negligible dipolar interactions. The atomic force microscopy and the scanning electron microscopyimages indicate that the patterned dots have well defined circular shape down to $400 \mathrm{~nm}$ diameter. Magnetic force microscopy (MFM) was used to study the magnetic domain structures of the samples.

In order to form single bubble state, we tested various field-induced demagnetization methods. In figure 1, we show MFM images obtained on1.25 $\mu$ mdiameter circular dots made of $[\mathrm{Co}(0.2) / \mathrm{Ni}(0.6)]_{10}$ multilayers after AC out-of-plane field demagnetization (figure 1(a)), single in-plane field application (figure 1(b)) and AC in-plane field demagnetization (figure 1(c)). We define the starting (i.e. maximum) field amplitude during the demagnetization process as $H_{\max }$. For all types of AC demagnetizations, samples were first saturated out-of-plane, then the field sweep rate was kept as $10 \mathrm{mT} / \mathrm{s}$ with maximum field decreasing by $0.3 \mathrm{mT}$ at each period. Out-of-plane AC magnetic field provides only single domain state, i.e. saturated state, with dot moments randomly pointing up or down (figure1(a)). The absence of checker board phase confirms negligibledipolar interactionsamongthe dots with $5 \mu \mathrm{m}$ center-to-center spacing. For comparison, full film with same composition and AC out-of-plane demagnetization also leads to a uniformly magnetized state without any strip-out instability [21] because of the large quality factor of our sample, $Q=2 K_{U} / \mu_{0} M_{S}^{2}>2[6,25]$ (see for instance a typical magnetizationvs. of out-of-plane field experimental hysteresis loop in figure 1(d)). 

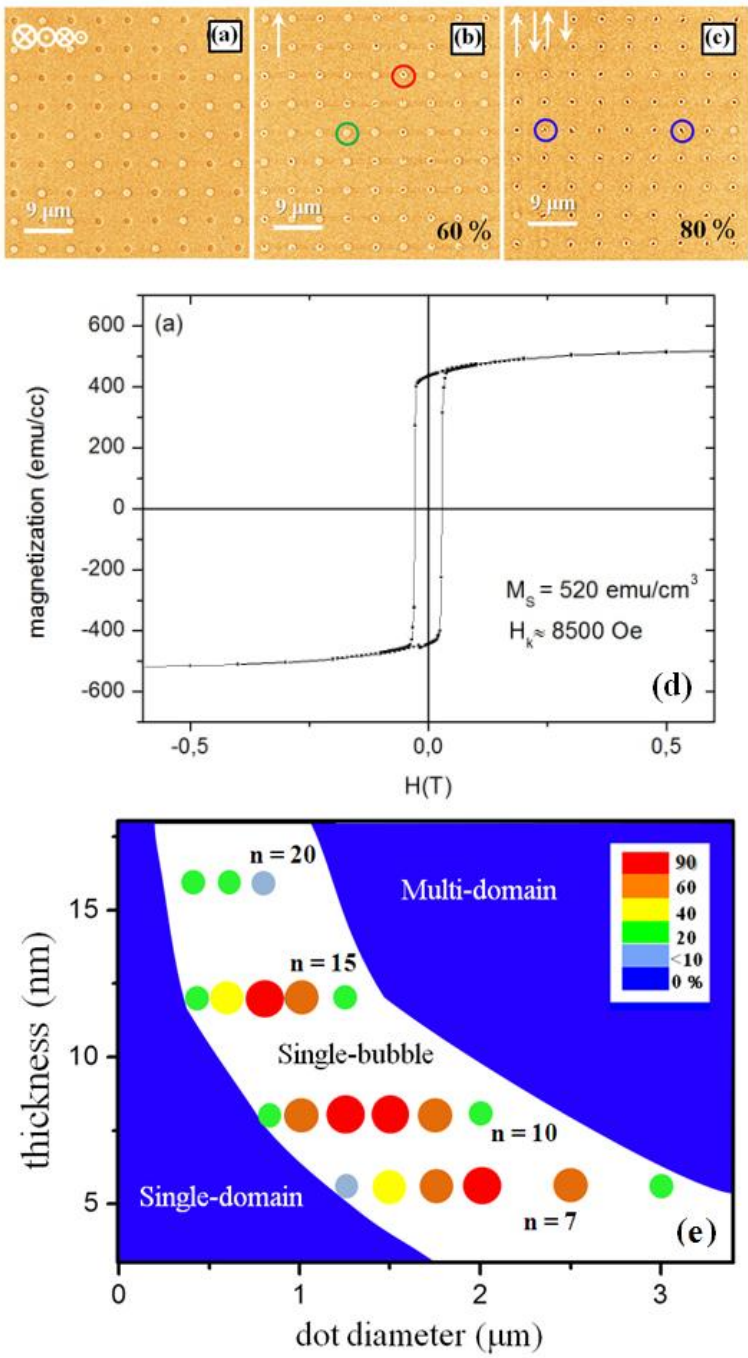

Figure 1.(a-c) MFM images of $45 \times 45 \mu \mathrm{m}^{2}$ array of $1.25 \mu \mathrm{m}$ diameter [Co/Ni] $]_{10}$ dots after demagnetization under (a) AC out-of-plane field, (b) one $0.4 \mathrm{~T}$ in-plane field pulse and (c) $\mathrm{AC}$ in-plane field starting from $H_{\max }=0.4 \mathrm{~T}$. The direction of the applied field is indicated in the left upper corner of the images. The percentage values indicate the ratio of single bubble state over 81 dots. The red, green, and blue circles show a single bubble state, single domain state (saturated state), and multi-domain states, respectively. (d) A typical magnetization vs outof-plane field experimental hysteresis loop for our full film samples. (e) Best percentage of single bubble as a function of the dots diameter and dot thickness for four $[\mathrm{Co} / \mathrm{Ni}]_{\mathrm{n}}$ multilayers with $\mathrm{n}=7,10,15$ and 20 , respectively. The color scale indicates the maximum percentage of single bubble, e.g. red corresponds to percentage between $60 \%$ and $90 \%$.

\section{RESULTS AND DISCUSSION}

Figure1(b) shows the MFM images of the dots' magnetic state after application of $0.4 \mathrm{~T}$ in-plane field. Magnetic bubbles(red circle in figure $1(\mathrm{~b})$ ) are observed in $60 \%$ of the dots while the others are still uniformly magnetized (green circle in figure1(b)). After a second field sweep in the opposite direction down to $-0.3985 \mathrm{~T}$ (not shown here), only $5 \%$ dots remained as single domain while $80 \%$ of the dots hold a well centered and circular bubble.Further AC demagnetization 
created a few more bubblenucleationsand made eachbubble slightly larger, more circular and well settled in the dot center. Figure1(c)corresponds to the magnetic configuration imaged after AC in-plane demagnetization starting at $0.4 \mathrm{~T}$. The last $15 \%$ of dots hold a magnetic configuration which consists of either more than one bubble or a bubble that is not centered and spreads to the dot edge (blue circles in figure 1(c)). We called these various states as "multi-domain state". Because it provides the best success rate, AC in-plane demagnetization was used in our further experiments.

In figure1(e), we plot an experimental bubble nucleation diagram, indicating the highest bubble percentage achieved experimentally after optimizing the demagnetization procedure (see below), as a function of multilayer thickness and dot diameter. The diagram shown in figure1(e) containsthree regions. The two blue regions correspond to the parametersspace where only single domain or multi-domain states are observed. Note that the concentric domain (see inset of figure2(d) and Refs. 22 and25) is experimentally rare in the multi-domain region and is usually reached neither experimentally nor in the micromagnetic simulations after in-plane AC demagnetization. The white region corresponds to parametersspace where the bubble can be formed and it is stable. By changing the number $n$ of $\mathrm{Co} / \mathrm{Ni}$ bilayer repeats, one may tune the thickness, i.e. the influence of demagnetization field, while keeping similar $K, M_{\mathrm{S}}$ and exchange strength $A$ [34]. As $n$ increases from 7 to 20 repeats the magnetostatic energy increases and, as a consequence, dot diameter has to be decreased in order to conserve single bubble formation [35].In general, for a fixed thickness, the dot diameter cannot be too small, otherwise the dipolar fields, coming from the dot volume and from the dot edge, are not large enough to compensate for the cost of bubble domain wall energy. No bubble can be stabilized and the dot remains uniformly magnetized (single domain state). On the other hand, if the dot diameter is too large, the average domain size is such that more than one bubble fit inside the dot (multi-domain state). Besides, as the dot thickness increases, the dipolar fields increase relative to the domain wall energy and the stable bubble diameter decreases. Therefore single bubble state and multi-domain state can be stabilized for smaller dot diameter in the case of larger thickness.Here, for comparison, the full film average domain width measured after in-plane AC demagnetization is about $300 \mathrm{~nm}$ at $n=10$ (not shown here). The phase diagram in figure1(e) is similar to those already reported in Refs. 22, 24 and 25, except that we select specific AC demagnetization procedure to achieve large percentage of bubble formation. 

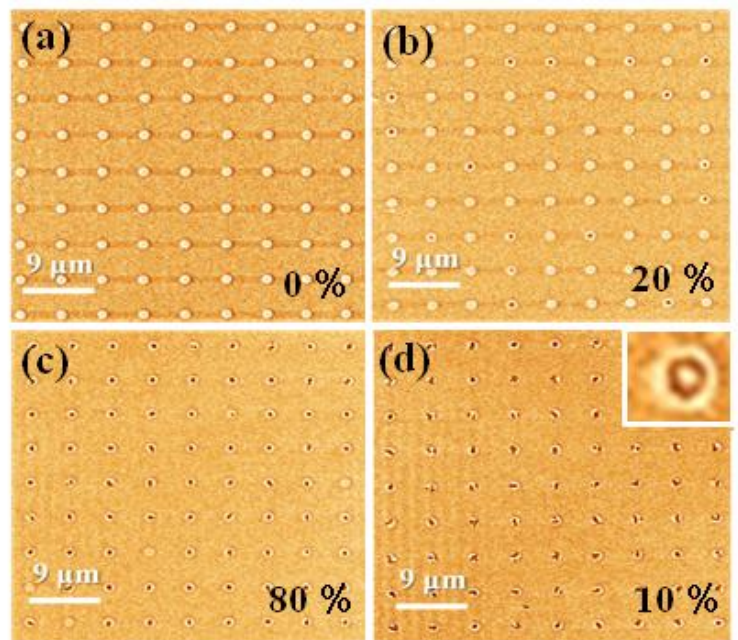

Figure 2.MFM images of $45 \times 45 \mu \mathrm{m}^{2}$ array of $1.25 \mu \mathrm{m}$ diameter[Co/Ni] $]_{10}$ dot array after $\mathrm{AC}$ demagnetization under in-plane field with $H_{\text {max }}$ of (a) $0.35 \mathrm{~T}$, (b) $0.375 \mathrm{~T}$, (c) $0.4 \mathrm{~T}$, and (d) $0.45 \mathrm{~T}$. Inset of (d) shows a concentric multidomain state in 1,75 $\mu \mathrm{m}$ diameter dot observed as a rare event. The percentagevalues indicate the ratio of single bubble state over 81 dots.

In order to further characterize the role of the demagnetization process on the formation of single domain, single bubble or multi-domain states, we demagnetized the same dot array as in figure 1by AC in-plane field starting from various $H_{\max }$ values. MFM images after demagnetization are shown in figure2(a-d). When $H_{\max } \leq 0.35 \mathrm{~T}$, all dots keeptheir initial single domain state, whereas $20 \%$ are in single bubble state for $H_{\max }=0.375 \mathrm{~T}$. At $H_{\max }=0.4 \mathrm{~T}, 5 \%$ of the dots remain single domain, $80 \%$ hold a bubble and $15 \%$ are in a multi-domain state. Further increase of $H_{\max }$ above $0.45 \mathrm{~T}$ leads to less than $10 \%$ of single domain and single bubble state and most of the dots are in multi-domain state. 

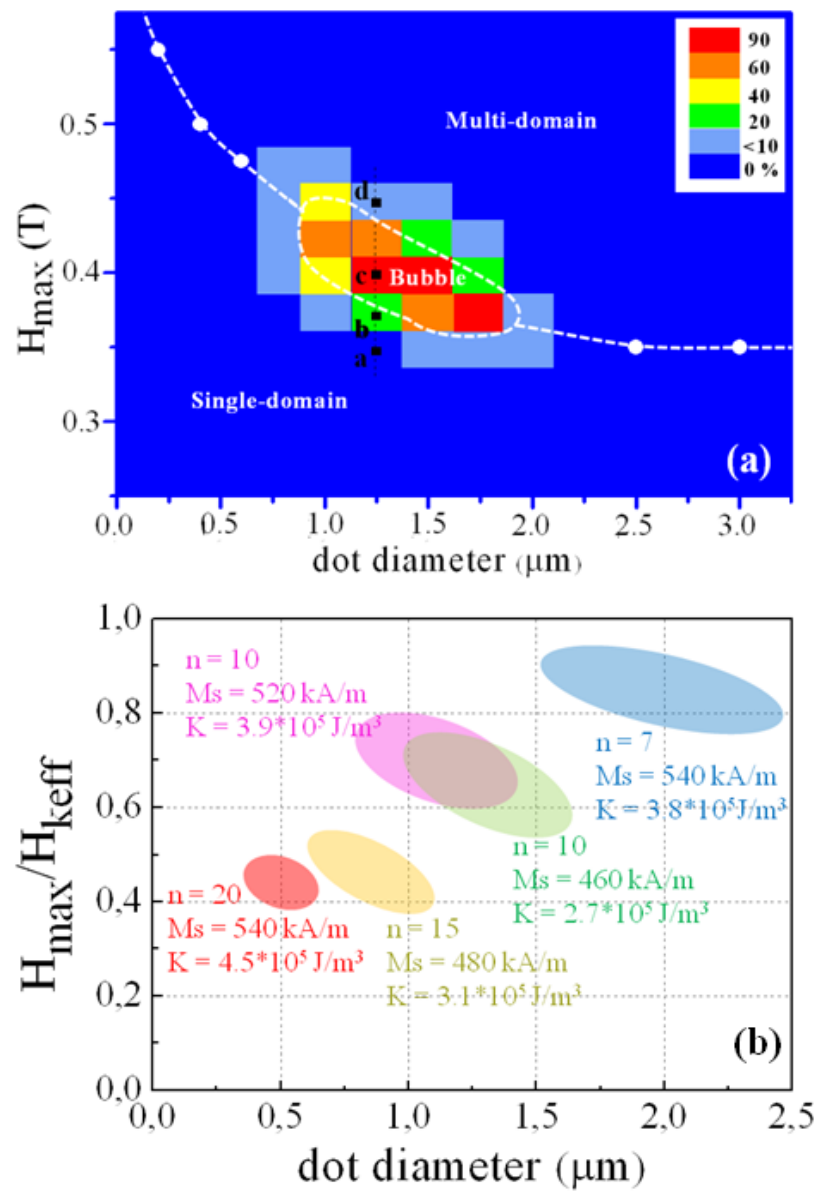

Figure 3.(a) Percentage of single bubble nucleation as a function of the dots diameter and $H_{\max }$ afterACdemagnetizationprocess for $[\mathrm{Co} / \mathrm{Ni}]_{10}$ dot array. The color code quantifies the percentage of single bubbles. The four black points marked as "a", "b", "c", and "d" are accordance with figure2(a), 2(b), 2(c), and 2(d), respectively. The color scale indicates the percentage of single bubble. (b) Zones of percentage of single-bubble higher than $10 \%$ as a function of $H_{\max }$ and dot diameter for Co/Ni MLs having $\mathrm{n}=7$ (blue), 10 (green and violet), 15 (orange), 20 (red) repeats. $M_{\mathrm{S}}$ and $K$ values are indicated for each sample.

We performed the same experiments as described in figure 2 for various dot diameters from 0.2 to $4 \mu \mathrm{m}$. We plot in figure3(a) a phase diagram indicating the limits of observation of the three magnetic states. As the dot diameter decreases, the value of $H_{\max }$ which marks the transition between the single domain and multi-domain state increases. A large percentage of bubble state is obtained only in a small region of" $H_{\max }$ vs. dot diameter" space. So we conclude that, in order to form large array of single bubbles, one has not only to tune the system parameters to ensure the energy metastability of the bubble as discussed, for instance, in Refs. 22, 24 and 25, but also to choose the right AC in-plane field demagnetization process. The same feature is observed for the five tested samples with different number of repeats from $n=7$ to 20. In figure3(b) we plot the region of reliable bubble formation (i.e. the circled region in figure 3(a)) as a function of dot diameter and $H_{\max } / H k_{\text {eff }}$ ratio 
for these five samples. Here $H k_{\text {effi }}$ is defined as " $2 K / M_{\mathrm{S}}-M_{\mathrm{S}}$ ". For accuracy we indicated on the figure3(b) the full film values of $M_{\mathrm{S}}$ and $K$ for each sample. For the same dot diameter, $H_{\mathrm{max}} / H k_{\mathrm{eff}}$ ratio required to obtain single bubble percentage decreases as $n$ increases.

Micromagneticcalculations were performed to understand the mechanism leading to each of the three states of the phase diagrams in figure3, depending on $H_{\max }$ amplitude of the AC demagnetizing process. We solved numerically the LandauLifshitz-Gilbert equation using three different micromagnetic codes based on finite difference, namely OOMMF [36], MUMAX3 [37], home-made solver [38]. Similar results have been obtained with the three solvers. We tested relaxation considering the equilibrium configuration achieved when the effective field energy and the magnetization are parallel for each computational cell (the algorithm stops for a residual down to $10^{-8}$ ) as well as time evolution.For the effective field, we take into account the exchange, self-magnetostatic and out-of-plane uniaxial anisotropy fields. For the simulations in figure 4 , we chose to simulate a $0.8 \mu \mathrm{m}$ diameter dot with $n=15$ repeats (i.e. $12 \mathrm{~nm}$ ) in order to limit computational time while maintaining discretization with $1.5 \times 1.5 \times 12 \mathrm{~nm}^{3}$ and $2 \times 2 \times 2 \mathrm{~nm}^{3}$ cells. The simulation parameters are exchange $A=1 \times 10^{-11} \mathrm{~J} / \mathrm{m}$, $M_{\mathrm{S}}=550 \mathrm{kA} / \mathrm{m}$ and anisotropy constant $K=3.3 \times 10^{5} \mathrm{~J} / \mathrm{m}^{3}$. We computed the magnetic configuration while an in-plane magnetic field wasset up to $H_{\max }$ and then decreased back to zero field. Three regimes could be observed depending on the value of $H_{\max }$ : (i) At $H_{\max }=0.2 \mathrm{~T}$, the dot magnetization is almost uniform and exhibits only a small tilt as compared with the perpendicular anisotropy axis (figure4(a)). As the field decreases from $H_{\max }$ towards remanence, the magnetic state remains uniform and the magnetization goes back along the anisotropy axis (figure $4(b, c)$ ). This process leading to the single-domain state is dominated by the exchange energy term. (ii) For intermediate values of $H_{\max }$ around $0.4 \mathrm{~T}$, the magnetic configuration exhibits a non-uniform spatial distribution of the $z$-component of the magnetization with both positive and negative region when applying $H_{\max }$ (figure4(d)). Indeed $H_{\max }$ larger than a threshold value (the so-called nucleation field) changes the energy landscape in such a way that the energy barrier that separates the uniform state from the other minima is decreased and the magnetostatic energy balances the exchange energy. In other words, when the in-plane field amplitude is at intermediate values, the system reduces its dipolar field energy by forming a wavy magnetic configuration (figure4(d)) with lateral variation of the $z$-component of the magnetization pointing successively in the up and down direction, instead of minimizing the exchange energy by maintaining all spins along a unique tilted direction as in figure1(b). Then, when the external field is reduced back towards zero, the demagnetization field freezes the magnetic configuration in a bubble state whose shape and diameter depend on the interplay between domain wall energy and dipolar energy. (iii) For $H_{\max }$ higher than $0.5 \mathrm{~T}$ (the socalled saturation field) the bubble state is no longer stable under in-plane $H_{\max }$ field and the magnetic configuration tends to become uniform with all moments pointing mostly along the in-plane field direction (figure4(h)). When the in-plane field is 
reduced, because of the symmetry of the magnetic configuration in figure4(i), the multi-domain state is then formed as a result of edge magnetic configuration (figure4(1)). The micromagnetic simulations successfully reproduce our experimental data. Similar calculation results have been achieved for dots with diameter of 0.5 and $1 \mu \mathrm{m}$. Based on the simulation findings, one can also understand the evolution of efficient $H_{\max } / H k_{\mathrm{eff}}$ with thickness for a given diameter dot. Indeed, as the thickness increases, the influence of the dipolar fields increases in the volume of the dot and helps to form the magnetic state in figure4(d)which further produces bubble at remanence. So the in-plane field required to form this magnetic configuration is decreased as $n$ increases.
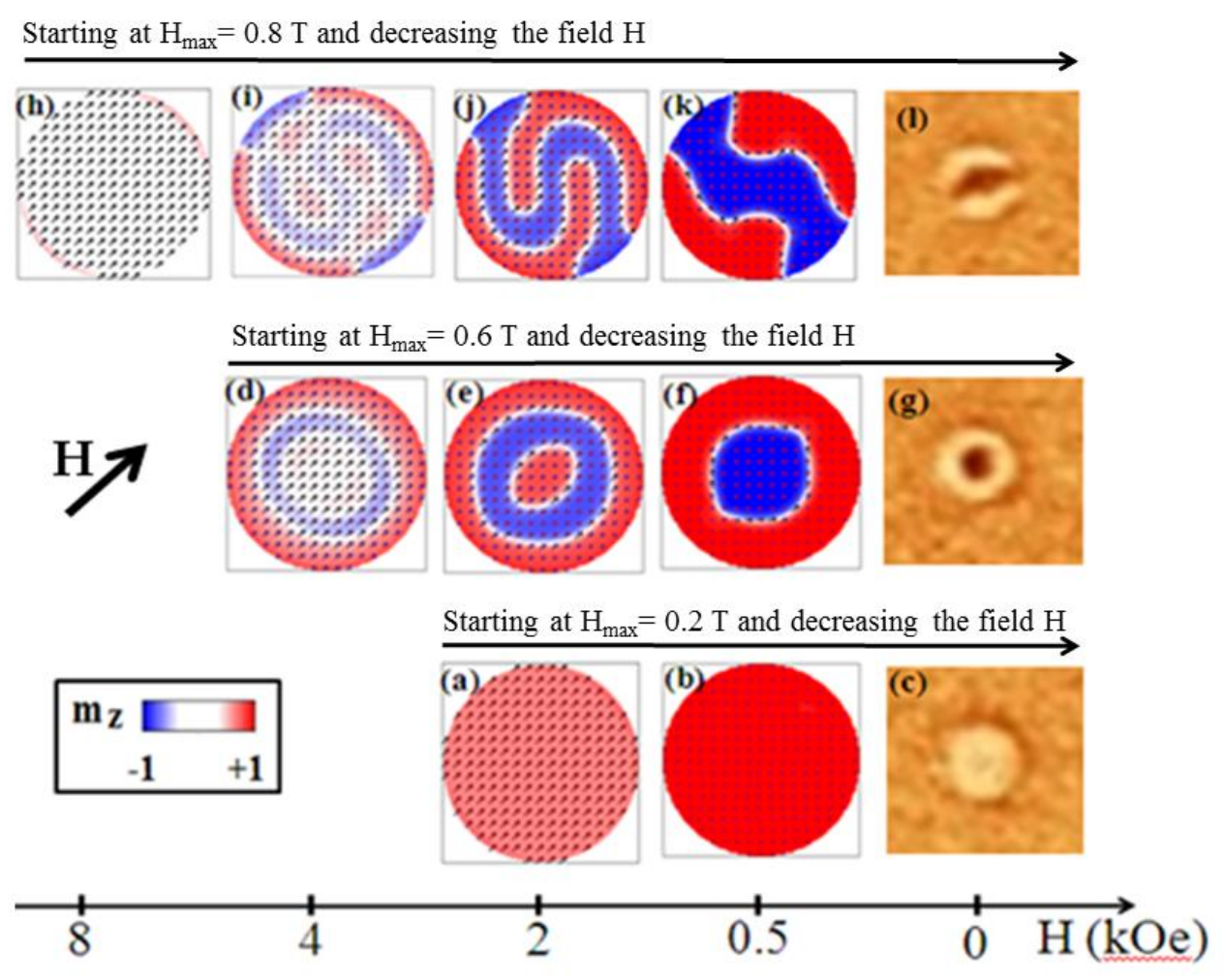

Figure 4. Snapshots of the calculated magnetic configuration in a $0.8 \mu \mathrm{m}$ diameter circular dot, having features of $[\mathrm{Co} / \mathrm{Ni}]_{15}$ sample, under in-plane applied field, starting from uniform out-of-plane state. The black arrow indicates the field $H$ direction. The three rows correspond to the three different values of $H_{\max }$ leading respectively to (i) uniform magnetic state if $H_{\max }$ is low, (ii) bubble nucleation for intermediate $H_{\max }$ value, and (iii) multi-domain if $H_{\max }$ is strong enough to almost saturate the dot in-plane; (c,g,l) are MFM images, corresponding to calculated states.

We would like to comment on the nature of the observed bubble equilibrium state. $M_{\mathrm{S}}$ and $K$ can beprecisely known from magnetrometry on full films, while the exchange constant is not easy to determine experimentally. Considering the 
experimental values of $550 \mathrm{kA} / \mathrm{m}$ for magnetizationand $3.3 \times 10^{5} \mathrm{~J} / \mathrm{m}$ for anisotropy, the bubble state has a lower energy than the monodomain state for exchange constant lower than $0.6 \times 10^{-11} \mathrm{~J} / \mathrm{m}$ and is no longer stable above $0.8 \times 10^{-11} \mathrm{~J} / \mathrm{m}$. In literature, an intermediate value between pure $\mathrm{Co}$ and pure $\mathrm{Ni}$ exchange constants such as $10^{-11} \mathrm{~J} / \mathrm{m}$ is generally considered to quantify $\mathrm{Co} / \mathrm{Ni}$ multilayers exchange[39, 40, 41, 42].Most probably the bubble state is a metastable state that can be stabilized experimentally by local $A$ or $K$ variations decreasing the domain wall energy (domain wall energy which provides a large part of the total energy). From the computed energy of the bubble state as a function of the bubble diameter (not shown), it is possible to estimate a minimum effective field $\mathrm{dE} / \mathrm{dMz}$ of about $5 \mathrm{mT}$ for an exchange constant of $10^{-11} \mathrm{~J} / \mathrm{m}$. Such a value is much lower than the typical values related to anisotropy distribution in perpendicular multilayer films [43].
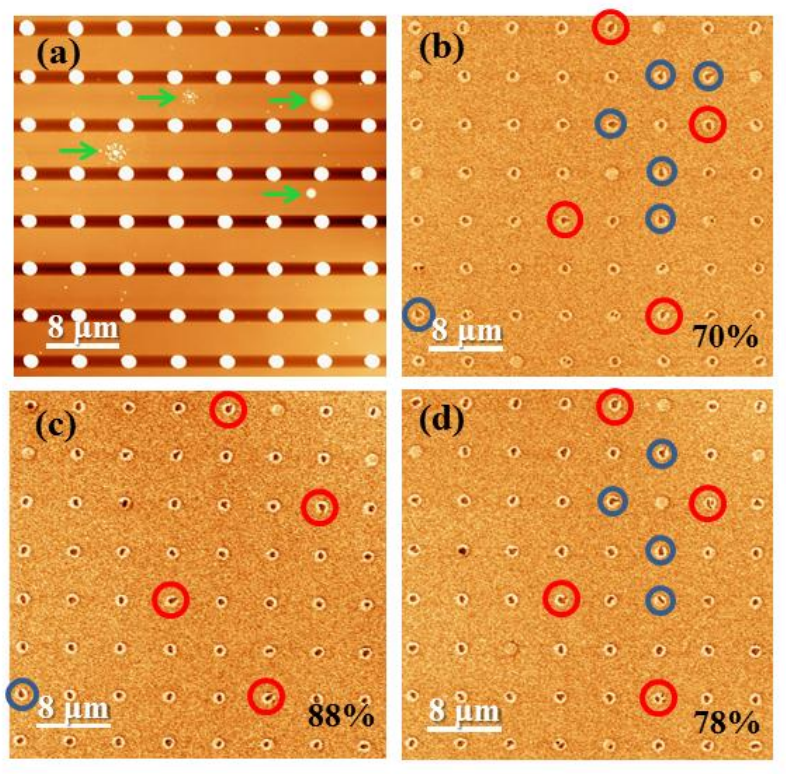

Figure 5. (a) AFM and (b-d) MFM images of $40 \times 40 \mu \mathrm{m}^{2}$ array of $1.25 \mu \mathrm{m}$ diameter[Co/Ni $]_{10}$ dot array after out-of-plane field saturation and then AC demagnetization under in-plane field with $H_{\max }$ of0.4T.The green arrow in (a) marks the defects used to locate the same region after each AC demagnetization. The percentagevalues in (b-d) indicate the ratio of single bubble state over 64 dots. The red circles indicate the dots containing a so-called broken bubble state, i.e. a bubble touching the dot edge, after each of the three AC demagnetizations (b-d). The blue circles indicate the dots containing a bubble touching the dot edge after two over three AC demagnetizations (b-d).

Finally, we tried to identify the reason why $100 \%$ success rate has never been reached during our experiments. Anisotropy and exchange distribution as well as the non-zero component of out-of-plane field during the in-plane AC demagnetization must play a role.In particular the polycrystalline nature of the sputtered $[\mathrm{Co} / \mathrm{Ni}]$ films often generates misorientated grain, grain boundaries and therefore anisotropy distribution [43] which could modify the magnetic 
processes presented in Fig. 4. Nevertheless the influence of dot edges has been found to be the main limitation to $100 \%$ success rate. To prove it, three AC demagnetization processes have been performed and MFM images have been taken after each. We used some non-magnetic defects between the dots to always image the same region of the sample. Figure5(b-d) shows between $70 \%$ and $88 \%$ of dots with a well centered single bubble, 4 to $5 \%$ of the dots are in uniform state and 8 to $16 \%$ contain a bubble that touches one region of the dot edge. We called this state "broken bubble state". In diagrams of figures1(e) and 3(a), it belongs to the more generic multi-domain state. Interestingly, the broken bubbles (when created) are always present in the same dots. The red circles or blue circles in figure5(b-d)indicate the dots containing broken bubble stateafter each of the threeor two over threeindependent AC demagnetizations, respectively. And even more interestingly, for each dot taken separately, the location at the edge region where the bubble touches the dot edge is the same after each AC demagnetization. Therefore, we can conclude that strong edge defects (probably due to patterning) induce nucleation in these dots or favor propagation of the bubble domain wall up to the edge. Improving the patterning process and removing edge defects would most probably allow us to reach bubble formation success rate close to $100 \%$.

\section{CONCLUSIONS}

Insummary, we studied the success rate of single magnetic bubble formation in an array of perpendicular anisotropy circular dots. Up to $90 \%$ of bubbles can be formed by tuning not only the dot and materials features, but also the bubble nucleation process. We demonstrate that higher success rate would be achieved by suppressing dot edge defects. We use an in-plane field AC demagnetization procedure whose success rate strongly depends on the starting field $H_{\text {max }}$ Micromagnetic calculations show thatit originates from the existence of a field range for which dipolar field favorsa wavy magnetic configuration (figure $4(\mathrm{~d})$ ) that transforms into single bubble state when the field is swept back towards remanence. Understanding how to form a single bubble state in a large array of nano-dots opens the path towards the characterization of bubbles response to rf-fields orspin-transfer torque (analogous to widely reported works on vortex) as well as towards

original magnonic crystals.In assembly of dots like magnonic crystal, reducing the distance between dots may affect the bubble nucleation process by increasing the dipolar fields interactions.

\section{ACKNOWLEDGMENTS}

The authors thank D. Lacour and S. Mc Murtry for help with respectively MFM and e-beam lithography experiments; thank DavidEllsworth and Jake Maclin for the help of English correction. This work was financially supported by FEDER, the Region Lorraine and the Grand Nancy and the bilateral agreement France-Turkey (TUBITAK-CNRS) project 
( TUBITAK Grant \#: 114F318,CNRS Grant \#PICS42452). The work of V.P. and G.F was supported by the project PRIN2010ECA8P3 from Italian MIUR.G.F and O.O. were also supported by the bilateral agreement Italy-Turkey (TUBITAK-CNR) project (CNR Grant \#: B52I14002910005, TUBITAK Grant \# 113F378) "Nanoscale magnetic devices based on the coupling of Spintronics and Spinorbitronics". 


\section{REFERENCES}

[1] Vukadinovic N and Boust F 2007 Phys. Rev. B75 014420

[2] Makhfudz I, Kruger B andTchernyshyov O 2012Phys. Rev. Lett.109 217201

[3] Sampaio J, Cros V, Rohart S, Thiaville A and Fert A 2013Nat. Nanotech.8 839

[4] Kim J-V, Garcia-Sanchez F, Sampaio J, Moreau-Luchaire C, Cros V and Fert A 2014 Phys. Rev. B90 064410

[5] Finocchio G, Ricci M, Tomasello R, Giordano A, Lanuzza M, Puliafito V Burrascano P, Azzerboni B and Carpentieri M 2015Appl. Phys. Lett.107262401

[6] Komineas S, Vaz C A F, Bland J A C and Papanicolau N 2005Phys. Rev. B (R)71060405

[7] Puliafito V, Torres L, Ozatay O, Hauet T, Azzerboni B and Finocchio G 2014 J. Appl. Phys.11517D139

[8] Moutafis C, Komineas S and Bland J A C 2009 Phys. Rev. B 79224429

[9] Guslienko K Y 2015 IEEE Magn.Lett.6 4000104

[10] Buttner F et al 2015 Nature Phys.11 225

[11] Iwasaki J, Mochizuki M and Nagaosa N 2013 Nat. Nanotech. 8742

[12] Tomasello R, Martinez E, Zivieri E, Torres L, Carpentieri M and Finocchio G 2014 Sci. Rep.4 6784

[13] Jiang W et al 2015 Science349 283

[14] Moreau-Luchaire C et al 2016 Nat. Nanotech.(published online);Boulle O et al 2016 Nat. Nanotech.(published online); Woo $\mathrm{S}$ et al 2015 arXiv:1502.07376v1

[15] Dzyaloshinskii I 1958 J. Phys. Chem. Solids4 241

[16] Moriya T 1960 Phys. Rev. Lett.4 228

[17] Choi J, Wu J, Won C, Wu Y Z, Scholl A, Doran A, Owens T and Qiu Z Q 2007Phys. Rev. Lett.98 207205

[18] Ozatay O, Hauet T, Florez S H, Katine J A, Moser A, Thiele J-U, Folks L and Terris B D 2009Appl. Phys. Lett.95 172502

[19] Mohseni S M et al 2013Science339 1259

[20] Macià F, Backes D and Kent A D 2014Nat. Nanotech.9992

[21] Bran C, Butenko A B, Kiselev N S, Wolff U, Schultz L, Hellwig O, Rößler U K, Bogdanov A N and Neu V 2009 Phys. Rev. B79 024430

[22] Hehn M, Ounadjela K, Bucher J-P, Rousseaux F, Decanini D, Bartenlian B andChappert C 1196 Science272 1782

[23] Eames P and Dahlberg E D 2002J. Appl. Phys.91 7986

[24] Skidmore G D, Kunz A, Campbell C E and Dahlberg E D 2004Phys. Rev. B70 012410

[25] Moutafis C, Komineas S, Vaz C A F, Bland J A C, Shima T, Seki T and Takanashi K 2007Phys. Rev. B76 104426

[26] Pribiag V S, Krivorotov I N, Fuchs G D, Braganca P M, Ozatay O, Sankey J C, Ralph D C andBuhrman R A 2007Nature Phys.3 498

[27] Finocchio G, Puliafito V, Komineas S, Torres L, Ozatay O, Hauet T and Azzerboni B 2013 J. Appl. Phys.114 163908

[28] Puliafito V, Siracusano G, Azzerboni B and Finocchio G 2014 IEEE Magn. Lett.5 3000104

[29] Girod S, Gottwald M, Andrieu S, McCord J, Mangin S, Fullerton E, Beaujour J M L, Krishnatreya B J and Kent A D 2009 Appl. Phys. Lett.94 262504

[30] Mangin S, Henry Y, Ravelosona D, Katine J A and Fullerton E E 2009 Appl. Phys. Lett.94 012502

[31] Bernstein D P et al 2011 Phys. Rev. B (R)83 180410

[32] Gimbert F, Calmels L and Andrieu S 2011 Phys. Rev. B84 094432

[33] Gottwald M et al 2012 Phys. Rev. B86 014425

[34] Hellwig O, Berger A, Kortright J B and Fullerton E E 2007 J. Magn.Magn.Mater.319 13

[35] Seul M and Andelman D 1995 Science267 476

[36] Donahue M J and Porter D G 1999 OOMMF User's Guide, Version 1.0, Interagency Report NISTIR 6376, Tech. Rep.(National Institute of Standards and Technology, Gaithersburg, MD)

[37] Vansteenkiste A, Leliaert J, Dvornik M, Helsen M, Garcia-Sanchez F and Van Waeyenberge B 2014 AIP Advances4 107133

[38] Giordano A, Finocchio G, Torres L, Carpentieri M and Azzerboni B 2012J. Appl. Phys.11107D112

[39] Tannenwald P E 1961 Phys. Rev.121 715

[40] Beaujour J M, Chen W, Krycka K, Kao C C, Sun J Z and Kent A D 2007 Europ. Phys. J. B59 475

[41] Ryu K, Thomas L, Yang S and Parkin S 2013 Nat. Nanotech.8 527533

[42] Hata H, Taniguchi T, Lee H-W, Moriyama T and Ono T 2014 Appl. Phys. Express. 7033001

[43] Pfau B et al 2011Appl. Phys. Lett.99 062502 